\title{
Characterization of the human SLC30A8 promoter and intronic enhancer
}

\author{
Lynley D Pound, Suparna A Sarkar ${ }^{1}$, Stéphane Cauchi², Yingda Wang, James K Oeser, \\ Catherine E Lee ${ }^{1}$, Philippe Froguel ${ }^{2,3}$, John C Hutton ${ }^{1}$ and Richard M O'Brien
}

Department of Molecular Physiology and Biophysics, Vanderbilt University School of Medicine, 8415 MRB IV, 2213 Garland Avenue, Nashville, Tennessee 37232-0615, USA

${ }^{1}$ Barbara Davis Center for Childhood Diabetes, University of Colorado at Denver, Aurora, Colorado 80045, USA

${ }^{2}$ UMR CNRS 8199, Genomics and Metabolic Diseases, 1 Rue du Professeur Calmette, 59019 Lille Cedex, France

${ }^{3}$ Department of Genomics of Common Diseases, Imperial College London, Hammersmith Hospital, Du Cane Road, London W12 0NN, UK

(Correspondence should be addressed to R M O’Brien; Email: richard.obrien @ vanderbilt.edu)

\begin{abstract}
Genome-wide association studies have shown that a polymorphic variant in SLC30A8, which encodes zinc transporter-8, is associated with altered susceptibility to type 2 diabetes (T2D). This association is consistent with the observation that glucose-stimulated insulin secretion is decreased in islets isolated from Slc30a8 knockout mice. In this study, immunohistochemical staining was first used to show that $S L C 30 A 8$ is expressed specifically in pancreatic islets. Fusion gene studies were then used to examine the molecular basis for the islet-specific expression of SLC30A8. The analysis of SLC30A8-luciferase expression in $\beta$ TC-3 cells revealed that the proximal promoter region, located between -6154 and -1 , relative to the translation start site, was only active in stable but not transient transfections. VISTA analyses identified three regions in the SLC30A8 promoter and a region in SLC30A8 intron 2 that are conserved in the mouse SIc30a8 gene. Additional fusion gene experiments demonstrated that none of these Slc30a8 promoter regions exhibited enhancer activity when ligated to a heterologous promoter whereas the conserved region in SLC30A8 intron 2 conferred elevated reporter gene expression selectively in $\beta$ TC-3 but not in $\alpha \mathrm{TC}-6$ cells. Finally, the functional effects of a single nucleotide polymorphism (SNP), rs62510556, in this conserved intron 2 enhancer were investigated. Gel retardation studies showed that rs62510556 affects the binding of an unknown transcription factor and fusion gene analyses showed that it modulates enhancer activity. However, genetic analyses suggest that this SNP is not a causal variant that contributes to the association between SLC30A8 and T2D, at least in Europeans.
\end{abstract}

Journal of Molecular Endocrinology (2011) 47, 251-259

\section{Introduction}

Zinc transporter-8 (ZnT-8) belongs to a family of zinc transporters that, along with metallothioneins, are involved in intracellular zinc homeostasis and signaling through compartmentation of the ion and regulation of its concentration (Wijesekara et al. 2009). The mouse Slc30a8 gene, which encodes ZnT-8, is mainly expressed in pancreatic $\alpha$ and $\beta$ cells (Gyulkhandanyan et al. 2008, Pound et al. 2009), with much lower levels of expression in testis and submaxillary glands (Murgia et al. 2009). ZnT-8 is localized to insulin secretory granules within $\beta$ cells (Chimienti et al. 2006) where it is thought to be required for providing zinc to allow for proper maturation, storage, and secretion of insulin (Wijesekara et al. 2009).

In keeping with an important role for ZnT-8 in the $\beta$ cell, recent genome-wide association (GWA) studies linked a non-synonymous single nucleotide polymorphism (SNP), rs13266634, which changes amino acid 325 of human ZnT-8 from tryptophan to arginine, to increased susceptibility to type 2 diabetes (T2D; Saxena et al. 2007, Scott et al. 2007, Sladek et al. 2007, Zeggini et al. 2007), gestational diabetes (Lauenborg et al. 2009), impaired proinsulin to insulin conversion (Stancakova et al. 2009), and reduced first-phase insulin secretion (Stancakova et al. 2009). This same variant is also associated with autoantibody epitope specificity changes in human type 1 diabetes (Wenzlau et al. 2008).

The rs13266634 variant has the potential to be causative, rather than just being in strong linkage disequilibrium with the actual causative variant, since it has been reported to affect zinc transport by ZnT-8 in cultured cell studies (Nicolson et al. 2009). However, a key question that remains to be addressed is whether this SNP is the only variant in the SLC30A8 gene altering susceptibility to T2D. This seems unlikely because the variants identified to date can only explain a small fraction of the calculated genetic contribution to the risk of developing T2D (Manolio et al. 2009). 
While various explanations have been proposed for this missing heritability (Manolio et al. 2009), one possibility is that rare SNPs, which are not analyzed in GWA studies, exist in genes such as SLC3OA8 that markedly affect their expression or the function of the encoded protein. The studies described here were initiated with the goal of characterizing key regions of the SLC30A8 gene so as to establish a framework for the future analysis of rare SNPs that have the potential to modulate SLCBOA8 gene transcription. We show that the proximal human SLC3OA8 promoter, located between -6154 and -1 , relative to the translation start site, is only active in stable and not transient transfections. In addition, we identify a conserved islet $\beta$ cell-specific enhancer in intron 2 of the SLC3OA8 gene. Finally, a SNP, rs62510556, in this conserved intron 2 enhancer is shown to affect the binding of an unknown transcription factor and thereby modulate enhancer activity. This SNP therefore had the potential to be a second causal variant that contributed to the association between SLC30A8 and T2D. However, genetic analyses suggest that, in Europeans, this SNP is not associated with the risk for T2D.

\section{Materials and methods}

\section{Materials}

The BAC plasmids from which mouse Slc30a8 and human SLC30A8 genomic regions were isolated (see below) were purchased from BACPAC Resources (Children's Hospital Oakland Research Institute, Oakland, CA, USA).

\section{Immunohistochemistry}

Human pancreata were fixed in $4 \%$ paraformaldehyde in PBS (Invitrogen Corporation) for $12 \mathrm{~h}$ and were paraffin embedded. Immunostainings were performed on $6 \mu \mathrm{m}$ sections that were de-paraffinized three times with Histoclear (National Diagnostics, Atlanta, GA, USA) for $5 \mathrm{~min}$, then hydrated serially in 100 (twice), 95, and $70 \%$ ethanol and water for $2 \mathrm{~min}$. Antigen retrieval was performed on sections by incubating in $0 \cdot 01 \mathrm{M}$ citrate buffer, $\mathrm{pH} 6 \cdot 0$, for $5 \mathrm{~min}$ in a microwave using medium power settings. Sections were incubated for $1 \mathrm{~h}$ at room temperature with blocking buffer (TSA system; Invitrogen Corporation) and then at room temperature overnight in a humid chamber with three primary antibodies: mouse anti-human ZnT-8 (1:20; John Hutton, UC Denver; please see Supplementary Data, see section on supplementary data given at the end of this article, for details on antibody generation), Guinea pig anti-insulin (1:50; Sigma), and rabbit antiglucagon (1:50; Dako North America, Inc., Carpinteria,
CA, USA) in TNB antibody dilution buffer (Invitrogen Corporation). The slides were then drained and washed three times for $5 \mathrm{~min}$ in PBS before secondary antibodies (1:250) conjugated to aminomethylcoumarin acetate, Cyanine 2, or Cyanine 5 fluorophores (Jackson Immunoresearch Laboratories, West Grove, PA, USA) were applied and incubated at room temperature for $60 \mathrm{~min}$. The slides were then washed in PBS and mounted with a glycerol-based media. Images were acquired with Intelligent Imaging System software using an Olympus 1X81-inverted-motorized microscope equipped with Olympus DSU spinning disk confocal optics and a Hamamatsu ORCA IIER monochromatic CCD camera. The images were later pseudo colored for illustration: red (ZnT-8), blue (glucagon), and green (insulin).

\section{Fusion gene plasmid construction}

Please see Supplementary Data, see section on supplementary data given at the end of this article.

\section{Cell culture, transfection, and luciferase assays}

Mouse islet $\beta$ cell-derived $\beta$ TC-3 cells and $\alpha$ cell-derived $\alpha$ TC-6 cells were grown in DMEM containing $10 \%$ (vol/vol) fetal bovine serum. For transient transfections, cells were transfected with $0 \cdot 5 \mu \mathrm{g}$ of an expression vector encoding SV40-Renilla luciferase (Promega) and $2 \mu \mathrm{g}$ of a firefly luciferase pGL3 or pGL4 fusion gene plasmid using the lipofectamine reagent (GibcoBRL) as described previously (Bischof et al. 2001). Following overnight incubation in serum-containing medium, cells were harvested by trypsin digestion and then solubilized in passive lysis buffer (Promega). After two cycles of freeze/thawing, firefly and Renilla luciferase activity were assayed using the Promega Dual-Luciferase Reporter Assay System according to the manufacturer's instructions. To correct for variations in transfection efficiency, the results are expressed as the ratio of firefly:Renilla luciferase activity. In addition, three independent preparations of each fusion gene plasmid construct were analyzed in triplicate.

For stable transfections, cells were transfected with $2 \mu \mathrm{g}$ of a firefly luciferase pGL4 fusion gene plasmid using the lipofectamine reagent as described previously (Bischof et al. 2001). Three independent preparations of each fusion gene plasmid construct were analyzed in triplicate. Following overnight culture at $37^{\circ} \mathrm{C}$, cells were incubated with serum-containing medium supplemented with hygromycin (Sigma-Aldrich) at a final concentration of $500 \mu \mathrm{g} / \mathrm{ml}$. Every 2-3 days, hygromycin-containing medium was replaced. After 3-4 weeks, or until individual colonies could be visualized, colonies were pooled using trypsinization, transferred 
to $10 \mathrm{~cm}$ dishes, and hygromycin treatment was discontinued. Once the cells had reached confluence, luciferase activity in the pooled cultures was then assessed and the Pierce BCA protein assay used to correct for variations in protein content.

\section{Statistical analyses}

The transfection data were analyzed for differences from the control values, as specified in the figure legends. Statistical comparisons were calculated using an unpaired Student's $t$-test. The level of significance was $P<0 \cdot 05$ (two-sided test).

\section{Results}

\section{The human SLC30A8 gene is expressed in both pancreatic islet $\alpha$ and $\beta$ cells}

Chimienti et al. (2004) had suggested that in pancreatic islets, expression of the mouse Slc30a8 gene, which encodes the zinc transporter $\mathrm{ZnT}-8$, is restricted to insulin-secreting $\beta$ cells, but recent data suggest that it is also expressed in glucagon-secreting $\alpha$ cells (Gyulkhandanyan et al. 2008, Pound et al. 2009). To assess the expression pattern of human SLC30A8, immunohistochemical staining was performed on human pancreas sections. Figure 1 shows that ZnT- 8 was absent from exocrine tissue but was detected in both $\alpha$ and $\beta$ cells in human pancreatic islets, consistent with observations made in mice (Gyulkhandanyan et al. 2008, Pound et al. 2009).

\section{Highly conserved putative human SLC30A8 and mouse SIc30a8 promoter regions are unable to drive reporter gene expression in transient transfection experiments}

To identify candidate regions of the human SLC30A8 and mouse Slc30a8 genes that may serve as promoters, a sequence alignment using the VISTA program (Frazer et al. 2004) was performed with human and mouse gene sequence $10 \mathrm{kbp} 5^{\prime}$ of the translation start site (Chimienti et al. 2004). This analysis identified several highly conserved regions in the mouse and human genes in the area located $\sim 8000$ and $6000 \mathrm{bp}$, respectively, $5^{\prime}$ of the translation start site (Fig. 2). Figure 2 also shows that, while the conserved regions are the same, the spacing between conserved regions is reduced in the human promoter. An analysis of SLC30A8-expressed sequence tags in the NCBI nucleotide database suggests that $S L C 30 A 8$ gene transcription initiates at multiple start sites in a $\sim 500 \mathrm{bp}$ region located immediately $5^{\prime}$ of the translation initiation codon, consistent with the absence of a consensus TATA box sequence in this location (Smale 1997). To investigate whether this region represents the SLC30A8 promoter, a SLC30A8-luciferase fusion gene was constructed, containing putative promoter sequence between -6154 and -1 , relative to the translation start site, and transiently transfected into $\beta$ TC-3 cells, an islet $\beta$ cell-derived cell line (Efrat et al. 1988). Basal luciferase expression directed by this fusion gene was below the level obtained with the promoterless luciferase expression vector (Fig. 3A). Identical results were obtained in transient transfection experiments in HIT
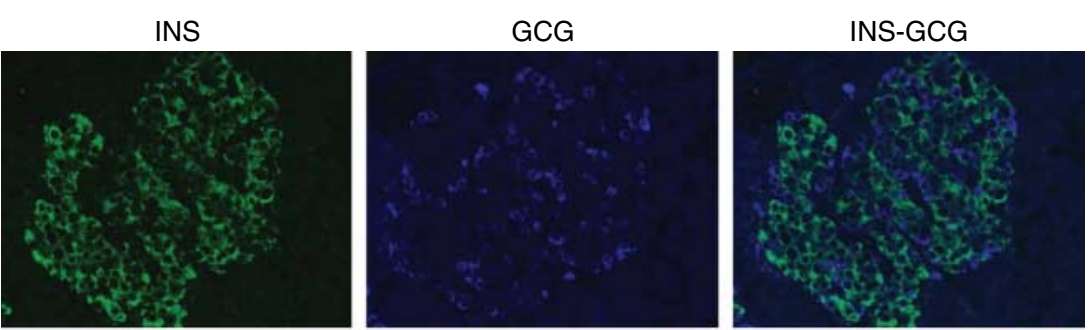

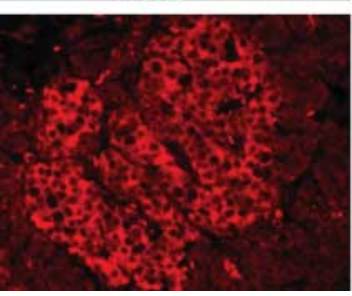

SLC30A8

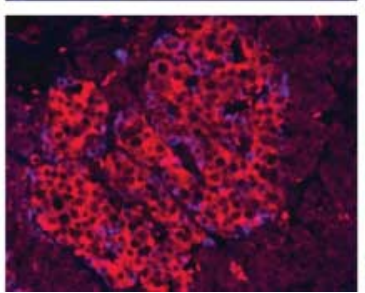

GCG-SLC30A8

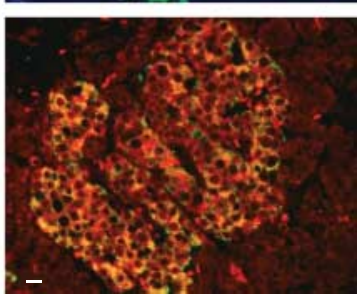

INS-SLC30A8

Figure 1 Immunohistochemical detection of $\mathrm{ZnT}-8$ in human pancreas. Paraffinembedded sections of adult human pancreata were immunostained for the presence of insulin (green) and glucagon (blue) and ZnT-8 (red) as described in the Materials and methods section. A representative section is shown. Scale bar: $10 \mu \mathrm{m}$. Full colour version of this figure available via http://dx.doi.org/10.1530/JME-11-0055. 


\section{A Human versus mouse}

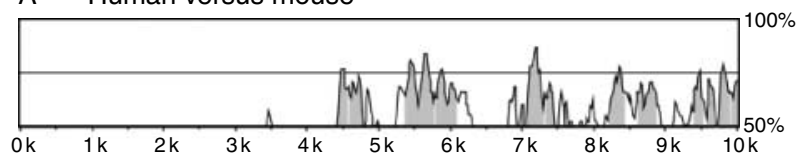

B Mouse versus human

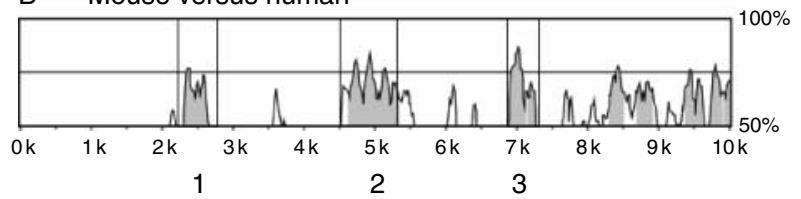

Figure 2 The putative human SLC30A8 and mouse SIC30a8 promoters are highly conserved. The figure shows a VISTA browser plot (Frazer et al. 2004) comparing $10 \mathrm{kbp}$ of human $S L C 30 A 8$ versus mouse S/c30a8 (A) and mouse S/c30a8 versus human SLC3OA8 (B) gene sequence. The translation start site is at bp 10000 and the level of conservation is indicated on the vertical axis. Conserved regions above $70 \% / 100 \mathrm{bp}$ are highlighted in gray and three putative promoter enhancer regions in the mouse SIC30a8 promoter are designated 1, 2, and 3 . It is shown that, while the conserved regions are the same, the spacing between conserved regions is reduced in the human promoter.

cells, an alternate islet $\beta$ cell-derived cell line, and with SLC30A8-luciferase fusion genes in both the pGL3 and the pGL4 luciferase vectors (data not shown). A Slc30a8-luciferase fusion gene containing putative mouse Slc30a8 promoter sequence between -1803 and -1 was also constructed and analyzed by transient transfection in both cells lines and in both luciferase vectors. As with the putative human promoter, the putative mouse promoter was also incapable of driving reporter gene expression (Fig. 3B and data not shown). The results of stable transfection experiments (see below) suggest that these human and mouse DNA fragments are not acting as repressors. Rather the reductions in reporter gene expression below the level obtained with the promoterless luciferase expression vector (Fig. 3A and B) are more likely due to the presence of SLC3OA8 and Slc30a8 genomic DNA further reducing low non-specific read-through transcription.

A possible explanation for the lack of promoter activity was that a cryptic RNA secondary structure, forming between the luciferase coding sequence and the $5^{\prime}$-untranslated region located between the transcription start site and translation start site, was impairing mRNA translation (Kozak 1989). Fusion genes were therefore generated containing genomic sequence that terminated at known human $(-6154$ / -235; NM_173851) and chimpanzee (-6154/-522; XM_519918) transcription start sites (Fig. 3A) or at a known mouse $(-1803 /-335$; NM_172816) transcription start site (Fig. 3B). However, none of these fusion genes conferred reporter gene expression in transient transfection assays (Fig. 3A and B).

\section{Identification of human SLC30A8 and mouse SIC30a8 promoter regions that drive reporter gene expression in stable transfection experiments}

Another possible explanation for the lack of fusion gene expression in transient transfection experiments was the known differences in chromatin structure between that on endogenous promoters and that on transiently transfected plasmid templates (Smith \& Hager 1997). Thus, previous studies have demonstrated the potential for major differences in expression levels when fusion genes are compared using transient and stable transfection (Smith \& Hager 1997). Some promoters/enhancers are only active, or are much more active, when analyzed in stable transfections (Smith \& Hager 1997). To assess whether chromatin structure was essential for human SLC3OA8 and mouse Slc30a8 promoter function, luciferase fusion genes containing either the human SLC30A8 sequence from -6154 to -1 or the mouse Slc30a8 sequence from -1803 to -1 were analyzed in the context of the pGL4 vector. This vector contains a hygromycin resistance gene, which allows for negative selection. $\beta$ TC-3 cells were stably transfected with these human or mouse promoter-luciferase fusion genes and treated with hygromycin until individual colonies were visualized. The individual colonies were then pooled and luciferase activity assayed. Under these conditions, the human SLC30A8 (Fig. 3C) and mouse Slc30a8 (Fig. 3D) genomic regions analyzed both drove fusion gene expression at much higher levels than the promoterless pGL4 vector, indicating the presence of functional promoters. A difference in activity was observed between the human and mouse promoters, but presumably this is mainly because the human promoter fragment used (Fig. 3C) was much longer than the mouse (Fig. 3D) and is likely to contain additional enhancer regions (Fig. 2). Although zinc has been reported to regulate $S l c 30 a 8$ gene expression in INS-1E cells (Smidt et al. 2009), no effect of zinc on human SLC30A8 promoter activity was observed in the stable cell lines (Supplementary Figure 1, see section on supplementary data given at the end of this article), suggesting that the effect of zinc is mediated through other genomic regions or post-transcriptionally.

The VISTA analysis identified three conserved regions in the mouse $5 l c 30 a 8$ promoter, located between approximately $(-7751$ and -7250$),(-5498$ and -4730$)$ and $(-3163$ and -2698$)$ (Fig. 2B). These regions are found in similar locations in the human SLC30A8 gene (Fig. 2A). We hypothesized that these regions might represent transcriptional enhancers important for islet-specific expression of the Slc30a8 gene. To address this hypothesis, these regions, designated as promoter enhancers 1, 2, and 3 (Fig. 2B), were isolated and ligated $5^{\prime}$ of a heterologous 
thymidine kinase (TK)-luciferase fusion gene containing $T K$ genomic sequence between -105 and +51 , relative to the transcription start site (Vander Kooi et al. 2005). Luciferase expression directed by these fusion genes was then analyzed by transient transfection of $\beta \mathrm{TC}-3$ cells. None of these Slc30a8 promoter regions enhanced reporter gene expression beyond that driven by the $T K$-luciferase fusion gene alone, regardless of orientation (Supplementary
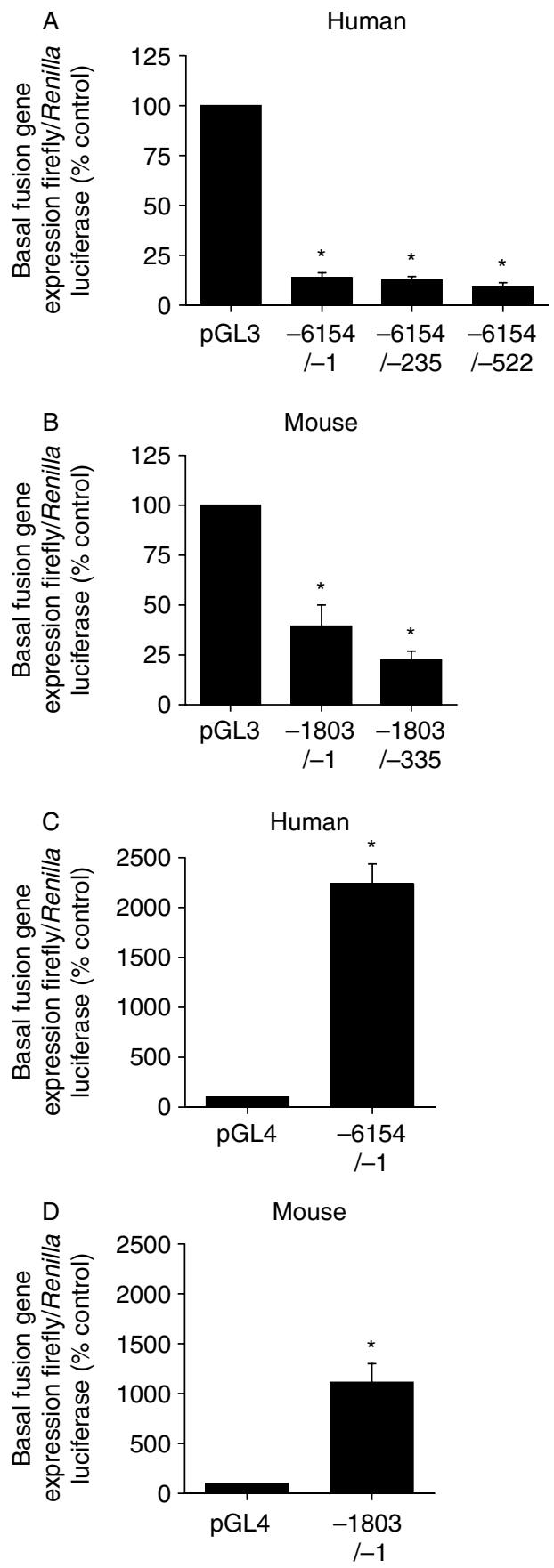

Figure 2, see section on supplementary data given at the end of this article). Similarly, when ligated $5^{\prime}$ of a heterologous G6PC2-luciferase fusion gene containing the proximal human $G 6 P C 2$ promoter sequence between -150 and +3 , again relative to the transcription start site, none of these Slc30a8 promoter regions enhanced reporter gene expression beyond that driven by the $-150 /+3$ G6PC2-luciferase fusion gene alone, regardless of orientation (Supplementary Figure 3, see section on supplementary data given at the end of this article). These data suggest that none of these promoter regions are enhancers.

\section{A conserved islet $\beta$ cell-specific enhancer is located in the second intron of the SLC3OA8 gene}

Intronic enhancers have previously been identified in several genes whose expressions are enriched in islets including those encoding glucagon (Zhou et al. 2006), islet amyloid polypeptide (Ekawa et al. 1997), and G6pc2 (Wang et al. 2008). We also recently identified an intronic enhancer located between +20125 and +20745 in intron 2 in the mouse Slc30a8 gene (Pound et al. 2010). A sequence alignment using the VISTA program shows that this region is conserved and found at a location between +16333 and +16954 in intron 2 in the human SLC3OA8 gene (Fig. 4).

Figure 3 The putative human SLC30A8 and mouse SIC30a8 promoter regions are able to drive reporter gene expression in stable but not transient transfection experiments. (A and B) $\beta T C-3$ cells were transiently co-transfected, as described in the Materials and methods section, with the empty pGL3 vector or the indicated human SLC30A8 (A) or mouse S/c30a8 (B) promoter-firefly luciferase fusion genes $(2 \mu \mathrm{g})$ and an expression vector encoding Renilla luciferase $(0.5 \mu \mathrm{g})$. Following transfection, cells were incubated for 18-20 $\mathrm{h}$ in serum containing medium and then harvested and luciferase activity assayed. Results are presented as the ratio of firefly:Renilla luciferase activity, expressed relative to the ratio obtained with the empty pGL3 vector, and represent the mean \pm S.E.M. of three experiments each using an independent preparation of all fusion gene plasmids with each plasmid assayed in triplicate. ${ }^{*} P<0 \cdot 05$. (C and D) $\beta T C-3$ cells were transfected with the empty pGL4 vector or the indicated human SLC30A8 (C) or mouse S/c30a8(D) promoter-firefly luciferase fusion genes $(2 \mu \mathrm{g})$. Following transfection, cells were incubated for 18-20 $\mathrm{h}$ in serum containing medium after which cells were incubated with media containing $500 \mu \mathrm{g} / \mathrm{ml}$ hygromycin. After 3-4 weeks, or until individual colonies could be visualized, colonies were pooled using trypsinization, transferred to $10 \mathrm{~cm}$ dishes, and hygromycin treatment was discontinued. Once the cells had reached confluence, luciferase activity in the pooled cultures was then assessed and the Pierce BCA protein assay used to correct for variations in protein content. Results are presented as the ratio of firefly activity to protein content in the cell lysate, expressed relative to the ratio obtained with the empty pGL4 vector, and represent the mean \pm S.E.M. of three experiments each using an independent preparation of all fusion gene plasmids with each plasmid assayed in triplicate. ${ }^{\star} P<0.05$. 


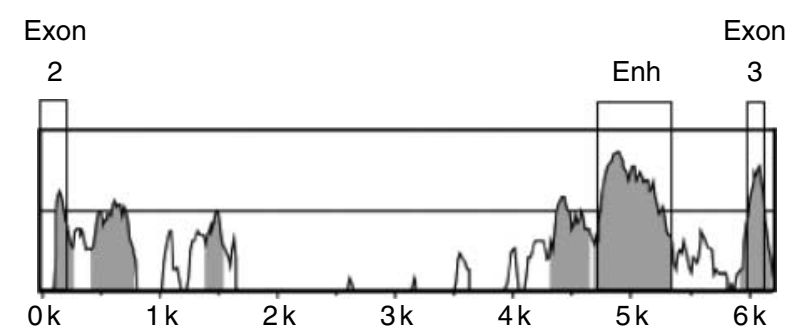

Figure 4 Identification of a highly conserved region within intron 2 of the human SLC30A8 and mouse SIc30a8 genes. A VISTA browser plot (Frazer et al. 2004) comparing the human SLC30A8 and mouse S/c30a8 gene sequences between exons 2 and 3 is shown. The level of conservation is indicated on the vertical axis. Conserved regions above $70 \% / 100 \mathrm{bp}$ are highlighted in gray. Exons 2 and 3 and the putative intron 2 enhancer (Enh) are boxed.

Fusion gene analyses showed that the mouse Slc30a8 enhancer in intron 2 was selectively active in $\beta$ TC-3 but not in $\alpha$ TC- 6 cells, indicating that this region is an islet $\beta$ cell-specific enhancer (Pound et al. 2010). To determine whether the +16579 and +16954 region of intron 2 in the human SLC3OA 8 gene also represents a transcriptional enhancer, this region was isolated using PCR and ligated $5^{\prime}$ of the $-150 /+3$ G6PC2luciferase fusion gene described above. Luciferase expression directed by this fusion gene was then analyzed by transient transfection of $\beta$ TC-3 and $\alpha$ TC- 6 cells. Figure 5A shows that, in $\beta$ TC-3 cells, this region elevated reporter gene expression beyond that driven by the $-150 /+3$ G6PC2-luciferase fusion gene alone. This effect was dependent on orientation (Fig. 5A), which is not consistent with the strict definition of an enhancer (Blackwood \& Kadonaga 1998). However, it is likely that, because of its close proximity to the G6PC2 promoter, this SLC30A8 enhancer is affecting transcription factor binding to the heterologous G6PC2 promoter in a manner that is different to the mechanism by which it affects SLC30A8 promoter activity from its native location in SLC30A8 intron 2. Strikingly, Fig. 5B shows that, in $\alpha$ TC- 6 cells, this region did not elevate reporter gene expression beyond that driven by the $-150 /+3$ G6PC2-luciferase fusion gene alone. These data demonstrate that this region is an islet $\beta$ cell-specific enhancer and suggest that the mechanisms controlling SLC30A8 expression in $\alpha$ and $\beta$ cells (Fig. 1) are overlapping but distinct.

\section{Discussion}

In this study, we show that the human SLC30A8 gene, like the mouse Slc30a8 gene, is expressed in both pancreatic islet $\alpha$ and $\beta$ cells (Fig. 1) and we identify a conserved SLC30A8 promoter region that drives fusion gene expression in stable, though not transient, transfections (Figs 2 and 3). Though it is uncommon for promoters/enhancers to require a precise chromatin context to function, several examples do exist (Smith \& Hager 1997). In addition, we have identified a conserved islet $\beta$ cell-specific enhancer in SLC30A8 intron 2 (Figs 4 and 5). A SNP, rs62510556, within this enhancer affects the binding of an unknown transcription factor and modulates enhancer activity (Supplementary Figure 4, see section on supplementary data given at the end of this article); however, genetic analyses suggest that, in Europeans, rs62510556 is not a causal variant that contributes to the association between SLC3OA8 and T2D (Supplementary Table 1, see section on supplementary data given at the end of this article). Future studies will examine whether an association exists in other populations. Thus, the
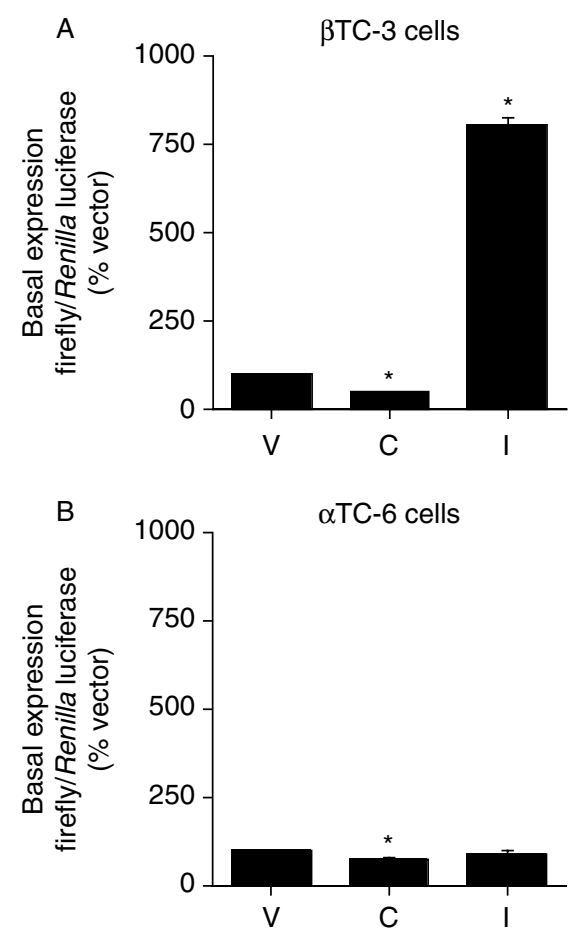

Figure 5 Analysis of the transcriptional enhancer activity of the highly conserved region within intron 2 of the human SLC30A8 gene in the context of the G6PC2 promoter. $\beta T C-3(A)$ and $\alpha$ TC-6 (B) cells were transiently co-transfected, as described in the Materials and methods section, with the indicated firefly luciferase fusion genes $(2 \mu \mathrm{g})$ and an expression vector encoding Renilla luciferase $(0.5 \mu \mathrm{g})$. The fusion genes represent the enhancerless human G6PC2 promoter-luciferase vector (V) or the SLC30A8 intron 2 sequence between +16579 and +16954 ligated to the human G6PC2 promoter-luciferase vector in the correct $(C)$ or inverted (I) orientation relative to the endogenous SLC30A8 gene. Following transfection, cells were incubated for $18-20 \mathrm{~h}$ in serumcontaining medium and then harvested and luciferase activity assayed. Results are presented as the ratio of firefly:Renilla luciferase activity, expressed relative to the ratio obtained with the enhancerless G6PC2 heterologous vector, and represent the mean \pm S.E.M. of three experiments each using an independent preparation of all fusion gene plasmids with each plasmid assayed in triplicate. ${ }^{*} P<0.05$. 
rs13266634 SLC30A8 variant, which changes amino acid 325 of human ZnT-8, is associated with T2D risk in Asians and Europeans, but not in Africans $(\mathrm{Xu}$ et al. 2011). Similarly, a variant, rs560887, in the G6PC2 gene has been associated with increased susceptibility to the development of T2D in some populations ( $\mathrm{Hu}$ et al. 2009, 2010, Reiling et al. 2009) but not others (Dupuis et al. 2010). These observations emphasize the importance of assessing the potential causative involvement of SNPs and genes in multiple populations.

While genetic data have strongly linked the SLC30A8 gene to increased susceptibility to the development of T2D in several, though not all, human populations $(\mathrm{Xu}$ et al. 2011), the manner in which defects in $\mathrm{ZnT}-8$ expression or function lead to islet dysfunction and hence diabetes is unclear. While the SLC30A8 rs13266634 variant has been reported to affect zinc transport by ZnT-8 in cultured cells (Nicolson et al. 2009), it does not affect glucose-stimulated insulin secretion from isolated human islets (Cauchi et al. 2010). However, this may simply relate to the small number of human islets examined (Cauchi et al. 2010) and the relatively small contribution of the SLC3OA 8 rs13266634 variant to diabetes risk (Saxena et al. 2007, Scott et al. 2007, Sladek et al. 2007, Zeggini et al. 2007). Surprisingly, though, studies in genetically modified mice, in which more significant changes in Slc30a8 expression can be achieved, have also failed to demonstrate a critical role for ZnT-8 in the control of glucose metabolism. Thus, mice in which the Slc30a8 gene was globally deleted have normal glucose tolerance (Lemaire et al. 2009, Nicolson et al. 2009, Pound et al. 2009) and mice with a $\beta$ cell-specific Slc30a 8 gene deletion have only mildly impaired glucose tolerance (Wijesekara et al. 2010). These observations suggest that either ZnT-8 is more important for the control of glucose metabolism in humans than mice or defects in glucose metabolism will only become apparent in older mice or mice harboring diseaseassociated variants in additional susceptibility genes. The latter possibilities appear more likely since the absence of ZnT-8 clearly affects $\beta$ cell function, as apparent from the observed reduction in fasting plasma insulin in mice lacking ZnT-8 as well as glucosestimulated insulin secretion and zinc content in islets isolated from those mice (Pound et al. 2009). Among these changes, the reduction in islet zinc content is quantitatively the most dramatic and also surprising, in relation to the mild metabolic phenotype observed, given the abundant literature supporting a key role of zinc in the proper maturation, storage, and secretion of insulin (Wijesekara et al. 2009). If future studies reveal that no association exists between rs62510556 and T2D, regardless of the population studied, this may indicate that the rs62510556 SNP does not change SLC30A8 expression sufficiently in vivo to have a biological effect or that the presence of a variant form of ZnT-8, as manifest by the rs13266634 SNP, increases T2D risk in some populations whereas changes in SLC30A8 expression do not. This last possibility is particularly intriguing given the very mild phenotype of Slc30a8 knockout mice since it would raise the interesting concept that perhaps it is the presence of a mutant form of ZnT-8 that causes problems with $\beta$ cell function whereas the absence of ZnT-8, as manifest in Slc30a8 knockout mice, is less deleterious.

The mild phenotype observed in Slc30a8 knockout mice may also be partly explained by the existence of unknown compensatory changes that have occurred during development to offset the absence of ZnT-8. Indeed, using an shRNA-mediated approach to downregulate Slc30a8 mRNA expression by $>90 \%$ in the INS-1 pancreatic $\beta$ cell line, Fu et al. (2009) demonstrated diminished uptake of exogenous zinc, reduced insulin content, and decreased glucose-stimulated insulin secretion. They also predicted that SNPs that affect SLC30A8 expression, such as the one reported here, would have the potential to affect insulin secretion and the glycemic response in vivo (Fu et al. 2009).

In summary, these experiments have shown that the human SLC30A8 genomic region located between -6154 and -1 , relative to the translation start site, contains a promoter that is only active in stable and not transient transfections. In addition, the human SLC30A8 genomic region located between +16579 and +16954 in intron 2 contains a conserved islet $\beta$ cell-specific enhancer. Given the critical role of ZnT-8 in islet zinc metabolism and the association between SLC3OA8 and T2D risk, it is possible that SNPs that affect SLC30A8 expression, rather than ZnT-8 function, may also influence T2D risk. While we find that a SNP, rs62510556, in the conserved intron 2 enhancer, which modulates enhancer activity, is not associated with T2D risk, at least in Europeans, it is possible that future studies may uncover rare SNPs with a more pronounced effect on SLC30A8 expression that do influence T2D risk.

\section{Supplementary data}

This is linked to the online version of the paper at http://dx.doi.org/ 10.1530/JME-11-0055.

\section{Declaration of interest}

The authors declare that there is no conflict of interest that could be perceived as prejudicing the impartiality of the research reported.

\section{Funding}

Research in the laboratory of R M O'B was supported by the NIH grants DK76027 and P60 DK20593 that supports the Vanderbilt 
Diabetes Center Core Laboratory. Research in the laboratory of J C H was supported by a Juvenile Diabetes Research Foundation Autoimmunity Prevention Center grant, NIH grants DK076027 and DK052068, and the Barbara Davis Center Diabetes and Endocrinology Research Center grant P30 DK57516. L D P was supported by the Vanderbilt Molecular Endocrinology Training Program grant 5T32 DK07563. S A S was supported by NIH grant K01DK080193 and JDRF grant 1-2008-1021.

\section{Acknowledgements}

We thank Shimon Efrat for providing the $\beta$ TC-3 and $\alpha$ TC- 6 cell lines.

\section{References}

Bischof LJ, Martin CC, Svitek CA, Stadelmaier BT, Hornbuckle LA, Goldman JK, Oeser JK, Hutton JC \& O'Brien RM 2001 Characterization of the mouse islet-specific glucose-6-phosphatase catalytic subunit-related protein gene promoter by in situ footprinting. Correlation with fusion gene expression in the islet derived bTC-3 and hamster insulinoma tumor cell lines. Diabetes 50 502-514. (doi:10.2337/diabetes.50.3.502)

Blackwood EM \& Kadonaga JT 1998 Going the distance: a current view of enhancer action. Science 281 60-63. (doi:10.1126/science.281. 5373.60)

Cauchi S, Del Guerra S, Choquet H, D'Aleo V, Groves CJ, Lupi R, McCarthy MI, Froguel P \& Marchetti P 2010 Meta-analysis and functional effects of the SLC30A8 rs13266634 polymorphism on isolated human pancreatic islets. Molecular Genetics and Metabolism 100 77-82. (doi:10.1016/j.ymgme.2010.01.001)

Chimienti F, Devergnas S, Favier A \& Seve M 2004 Identification and cloning of a beta-cell-specific zinc transporter, ZnT-8, localized into insulin secretory granules. Diabetes 53 2330-2337. (doi:10.2337/ diabetes.53.9.2330)

Chimienti F, Devergnas S, Pattou F, Schuit F, Garcia-Cuenca R, Vandewalle B, Kerr-Conte J, Van Lommel L, Grunwald D, Favier A et al. 2006 In vivo expression and functional characterization of the zinc transporter ZnT8 in glucose-induced insulin secretion. Journal of Cell Science 119 4199-4206. (doi:10.1242/jcs.03164)

Dupuis J, Langenberg C, Prokopenko I, Saxena R, Soranzo N, Jackson AU, Wheeler E, Glazer NL, Bouatia-Naji N, Gloyn AL et al. $2010 \mathrm{New}$ genetic loci implicated in fasting glucose homeostasis and their impact on type 2 diabetes risk. Nature Genetics 42 105-116. (doi:10. $1038 /$ ng.520)

Efrat S, Linde S, Kofod H, Spector D, Delannoy M, Grant S, Hanahan D \& Baekkeskov 1988 Beta-cell lines derived from transgenic mice expressing a hybrid insulin gene-oncogene. PNAS 85 9037-9041. (doi:10.1073/pnas.85.23.9037)

Ekawa K, Nishi M, Ohagi S, Sanke T \& Nanjo K 1997 Cloning of mouse islet amyloid polypeptide gene and characterization of its promoter. Journal of Molecular Endocrinology 19 79-86. (doi:10.1677/jme.0. 0190079)

Frazer KA, Pachter L, Poliakov A, Rubin EM \& Dubchak I 2004 VISTA: computational tools for comparative genomics. Nucleic Acids Research 32 W273-W279. (doi:10.1093/nar/gkh458)

Fu Y, Tian W, Pratt EB, Dirling LB, Shyng SL, Meshul CK \& Cohen DM 2009 Down-regulation of ZnT8 expression in INS-1 rat pancreatic beta cells reduces insulin content and glucose-inducible insulin secretion. PLoS ONE 4 e5679. (doi:10.1371/journal.pone.0005679)

Gyulkhandanyan AV, Lu H, Lee SC, Bhattacharjee A, Wijesekara N, Fox JE, MacDonald PE, Chimienti F, Dai FF \& Wheeler MB 2008 Investigation of transport mechanisms and regulation of intracellular $\mathrm{Zn}^{2+}$ in pancreatic alpha-cells. Journal of Biological Chemistry 283 10184-10197. (doi:10.1074/jbc.M707005200)
Hu C, Zhang R, Wang C, Ma X, Wang C, Fang Q, Bao Y, Xiang K \& Jia W 2009 A genetic variant of G6PC2 is associated with type 2 diabetes and fasting plasma glucose level in the Chinese population. Diabetologia 52 451-456. (doi:10.1007/s00125-008-1241-3)

Hu C, Zhang R, Wang C, Yu W, Lu J, Ma X, Wang J, Jiang F, Tang S, Bao Yet al. 2010 Effects of GCK, GCKR, G6PC2 and MTNR1B variants on glucose metabolism and insulin secretion. PLOS ONE 5 e11761. (doi:10.1371/journal.pone.0011761)

Kozak M 1989 The scanning model for translation: an update. Journal of Cell Biology 108 229-241. (doi:10.1083/jcb.108.2.229)

Lauenborg J, Grarup N, Damm P, Borch-Johnsen K, Jorgensen T, Pedersen O \& Hansen T 2009 Common type 2 diabetes risk gene variants associate with gestational diabetes. Journal of Clinical Endocrinology and Metabolism 94 145-150. (doi:10.1210/jc.20081336)

Lemaire K, Ravier MA, Schraenen A, Creemers JW, Van de Plas R, Granvik M, Van Lommel L, Waelkens E, Chimienti F, Rutter GA et al. 2009 Insulin crystallization depends on zinc transporter ZnT8 expression, but is not required for normal glucose homeostasis in mice. PNAS 106 14872-14877. (doi:10.1073/pnas.0906587106)

Manolio TA, Collins FS, Cox NJ, Goldstein DB, Hindorff LA, Hunter DJ, McCarthy MI, Ramos EM, Cardon LR, Chakravarti A et al. 2009 Finding the missing heritability of complex diseases. Nature $\mathbf{4 6 1}$ 747-753. (doi:10.1038/nature08494)

Murgia C, Devirgiliis C, Mancini E, Donadel G, Zalewski P \& Perozzi G 2009 Diabetes-linked zinc transporter ZnT8 is a homodimeric protein expressed by distinct rodent endocrine cell types in the pancreas and other glands. Nutrition, Metabolism, and Cardiovascular Diseases 19 431-439. (doi:10.1016/j.numecd.2008.09.004)

Nicolson TJ, Bellomo EA, Wijesekara N, Loder MK, Baldwin JM, Gyulkhandanyan AV, Koshkin V, Tarasov AI, Carzaniga R, Kronenberger $\mathrm{K}$ et al. 2009 Insulin storage and glucose homeostasis in mice null for the granule zinc transporter ZnT8 and studies of the type 2 diabetes-associated variants. Diabetes 58 2070-2083. (doi:10.2337/db09-0551)

Pound LD, Sarkar S, Benninger RK, Wang Y, Suwanichkul A, Shadoan MK, Printz RL, Oeser JK, Lee CE, Piston DW et al. 2009 Deletion of the mouse Slc30a8 gene encoding zinc transporter-8 results in impaired insulin secretion. Biochemical Journal421 371-376. (doi:10. 1042/BJ20090530)

Pound LD, Hang Y, Sarkar SA, Wang Y, Milam LA, Oeser JK, Printz RL, Lee CE, Stein R, Hutton JC et al. 2010 The pancreatic islet betacell-enriched transcription factor Pdx-1 regulates Slc30a8 gene transcription through an intronic enhancer. Biochemical Journal 433 95-105. (doi:10.1042/BJ20101488)

Reiling E, van 't Riet E, Groenewoud MJ, Welschen LM, van Hove EC, Nijpels G, Maassen JA, Dekker JM \& t Hart LM 2009 Combined effects of single-nucleotide polymorphisms in GCK, GCKR, G6PC2 and MTNR1B on fasting plasma glucose and type 2 diabetes risk. Diabetologia 52 1866-1870. (doi:10.1007/s00125-009-1413-9)

Saxena R, Voight BF, Lyssenko V, Burtt NP, de Bakker PI, Chen H, Roix JJ, Kathiresan S, Hirschhorn JN, Daly MJ et al. 2007 Genome-wide association analysis identifies loci for type 2 diabetes and triglyceride levels. Science 316 1331-1336. (doi:10.1126/science. 1142358)

Scott LJ, Mohlke KL, Bonnycastle LL, Willer CJ, Li Y, Duren WL, Erdos MR, Stringham HM, Chines PS, Jackson AU et al. 2007 A genomewide association study of type 2 diabetes in Finns detects multiple susceptibility variants. Science 316 1341-1345. (doi:10.1126/science. 1142382)

Sladek R, Rocheleau G, Rung J, Dina C, Shen L, Serre D, Boutin P, Vincent D, Belisle A, Hadjadj S et al. 2007 A genome-wide association study identifies novel risk loci for type 2 diabetes. Nature 445 881-885. (doi:10.1038/nature05616)

Smale ST 1997 Transcription initiation from TATA-less promoters within eukaryotic protein-coding genes. Biochimica et Biophysica Acta 1351 73-88. (doi:10.1016/S0167-4781(96)00206-0) 
Smidt K, Jessen N, Petersen AB, Larsen A, Magnusson N, Jeppesen JB, Stoltenberg M, Culvenor JG, Tsatsanis A, Brock B et al. 2009 SLC30A3 responds to glucose- and zinc variations in beta-cells and is critical for insulin production and in vivo glucose-metabolism during beta-cell stress. PLoS ONE 4 e5684. (doi:10.1371/journal. pone.0005684)

Smith CL \& Hager GL 1997 Transcriptional regulation of mammalian genes in vivo. A tale of two templates. Journal of Biological Chemistry 272 27493-27496. (doi:10.1074/jbc.272.44.27493)

Stancakova A, Kuulasmaa T, Paananen J, Jackson AU, Bonnycastle LL, Collins FS, Boehnke M, Kuusisto J \& Laakso M 2009 Association of 18 confirmed susceptibility loci for type 2 diabetes with indices of insulin release, proinsulin conversion, and insulin sensitivity in 5 327 non-diabetic Finnish men. Diabetes 58 2129-2136. (doi:10. 2337/db09-0117)

Vander Kooi BT, Onuma H, Oeser JK, Svitek CA, Allen SR, Vander Kooi CW, Chazin WJ \& O'Brien RM 2005 The glucose-6phosphatase catalytic subunit gene promoter contains both positive and negative glucocorticoid response elements. Molecular Endocrinology 19 3001-3022. (doi:10.1210/me.2004-0497)

Wang Y, Flemming BP, Martin CC, Allen SR, Walters J, Oeser JK, Hutton JC \& O'Brien RM 2008 Long-range enhancers are required to maintain expression of the autoantigen islet-specific glucose-6phosphatase catalytic subunit-related protein in adult mouse islets in vivo. Diabetes 57 133-141. (doi:10.2337/db07-0092)

Wenzlau JM, Liu Y, Yu L, Moua O, Fowler KT, Rangasamy S, Walters J, Eisenbarth GS, Davidson HW \& Hutton JC 2008 A common nonsynonymous single nucleotide polymorphism in the SLC30A8 gene determines ZnT8 autoantibody specificity in type 1 diabetes. Diabetes 57 2693-2697. (doi:10.2337/db08-0522)
Wijesekara N, Chimienti F \& Wheeler MB 2009 Zinc, a regulator of islet function and glucose homeostasis. Diabetes, Obesity $\mathcal{E}^{\circ}$ Metabolism 11 (Suppl 4) 202-214. (doi:10.1111/j.1463-1326.2009.01110.x)

Wijesekara N, Dai FF, Hardy AB, Giglou PR, Bhattacharjee A, Koshkin V, Chimienti F, Gaisano HY, Rutter GA \& Wheeler MB 2010 Beta cell-specific Znt8 deletion in mice causes marked defects in insulin processing, crystallisation and secretion. Diabetologia 53 1656-1668. (doi:10.1007/s00125-010-1733-9)

Xu K, Zha M, Wu X, Yu Z, Yu R, Xu X, Chen H \& Yang T 2011 Association between rs $13266634 \mathrm{C} / \mathrm{T}$ polymorphisms of solute carrier family 30 member 8 (SLC30A 8 ) and type 2 diabetes, impaired glucose tolerance, type 1 diabetes - a meta-analysis. Diabetes Research and Clinical Practice 91 195-202. (doi:10.1016/j. diabres.2010.11.012)

Zeggini E, Weedon MN, Lindgren CM, Frayling TM, Elliott KS, Lango H, Timpson NJ, Perry JR, Rayner NW, Freathy RM et al. 2007 Replication of genome-wide association signals in UK samples reveals risk loci for type 2 diabetes. Science 316 1336-1341. (doi:10.1126/science.1142364)

Zhou L, Nian M, Gu J \& Irwin DM 2006 Intron 1 sequences are required for pancreatic expression of the human proglucagon gene. American Journal of Physiology. Regulatory, Integrative and Comparative Physiology 290 R634-R641. (doi:10.1152/ajpregu. 00596.2005)

Received in final form 4 July 2011

Accepted 28 July 2011

Made available online as an Accepted Preprint 28 July 2011 